$03,10,12$

\title{
Расчеты из первых принципов колебательных спектров сверхрешеток CdSe/CdS
}

\author{
() А.И. Лебедев \\ Московский государственный университет им. М.В. Ломоносова, \\ Москва, Россия \\ E-mail: swan@scon155.phys.msu.ru
}

Поступила в Редакцию 30 июня 2021 г.

В окончательной редакции 4 июля 2021 г.

Принята к публикации 4 июля 2021 г.

Из первых принципов методом функционала плотности рассчитаны колебательные спектры сверхрешеток (CP) CdSe/CdS с различной толщиной слоев. Показано, что наряду со сложенными (folded) акустическими модами и ограниченными (confined) оптическими модами в СР возникает целый ряд ограниченных акустических мод, а в структурах с минимальной толщиной одного из слоев - также микроскопические интерфейсные моды типа локальной и щелевой мод. Анализ проекций собственных векторов колебательных мод в СР на ортонормированный базис нормальных колебаний в бинарных соединениях позволил установить особенности формирования этих колебательных мод и, в частности, определить степень перемешивания акустических и оптических колебаний. Сопоставление частот колебательных мод в СР и нанопластинках $\mathrm{CdSe} / \mathrm{CdS}$ позволило разделить влияние эффектов размерного квантования и релаксации поверхности на частоты колебаний в нанопластинках.

Ключевые слова: фононные спектры, полупроводниковые сверхрешетки, селенид кадмия, сульфид кадмия, наноструктуры.

DOI: 10.21883 /FTT.2021.12.51663.156

\section{1. Введение}

Методы колебательной спектроскопии - рамановское рассеяние и инфракрасное (ИК) поглощение представляют собой мощный инструмент для анализа свойств различных материалов. Эти методы нашли широкое применение при исследовании низкоразмерных структур [1-3]. Они позволяют получить информацию о реальной структуре исследуемых образцов: их составе, размерах, присутствующих в них механических деформациях, состоянии границ раздела и релаксации атомов вблизи поверхности. Однако интерпретация полученных результатов часто бывает недостаточно обоснованной. Задачей настоящей работы является анализ колебательных спектров сверхрешеток (CP) $\mathrm{CdSe} / \mathrm{CdS}$ с целью помочь в этой интерпретации. В результате численного моделирования спектров колебаний СР мы увидим ряд ранее мало обсуждавшихся колебательных мод, таких как ограниченные (confined) акустические колебания, щелевые и локальные моды. В отличие от большого числа предшествующих работ, в которых при расчете динамики решетки обычно использовались упрощенные модели, в настоящей работе для моделирования колебаний решетки используется метод функционала плотности, в котором электрические и механические граничные условия при релаксации структуры (материалы которой по параметру решетки различаются на $\sim 4 \%$ ) учитываются и удовлетворяются автоматически.

Исследования колебательных спектров сверхрешеток проводятся уже более 40 лет [4-26]. Уже в первых работах были установлены основные отличительные особенности колебательных спектров СР: возникновение в них сложенных (folded) акустических мод [4-6,10-14] и ограниченных (confined) оптических мод [8,10-14].

Сложенные моды представляют собой продольные акустические (LA) и поперечные акустические (TA) колебания, которые распространяются в обоих материалах, испытывая небольшие отражения на границах различающихся по акустическим свойствам материалов. Частота таких колебаний в двух материалах сверхрешетки одинакова, а волновые векторы - разные. Период сверхрешетки определяет новые периодические граничные условия возникновения стоячих волн, а тот факт, что минизона Бриллюэна сверхрешетки в несколько раз меньше размера зоны Бриллюэна в объемных материалах, приводит к тому, что эта зона „складывается“ в направлении роста так, что ряд точек из объема зоны Бриллюэна исходных материалов проецируются в Г-точку сложенной зоны. Таким образом, в СР возникает целый ряд новых колебательных мод в центре зоны Бриллюэна. Появление разрывов в значениях энергий мод (стоп-зон), наблюдаемых в центре и на границе минизоны Бриллюэна, связано с различием удельных акустических импедансов используемых материалов.

В области оптических колебаний в сверхрешетках наблюдаются ограниченные моды, в которых продольные оптические (LO) и поперечные оптические (TO) колебания локализованы в одном из материалов структуры и быстро затухают в другом материале. Для возникновения таких мод необходимо, чтобы колебания 
с данной частотой могли распространяться в одним из материалов и не могли распространяться в другом. Признаком таких колебаний является отсутствие дисперсии моды и сильная зависимость ее частоты от толщины слоя первого материала.

Наконец, при определенных условиях в сверхрешетках могут возникать интерфейсные (interface) моды моды, локализованные на границе раздела двух материалов, которые затухают в обоих материалах. Интерфейсные сильно локализованные моды (их еще называют микроскопическими) впервые были обнаружены в сверхрешетках $\mathrm{InAs} / \mathrm{GaSb}$ [27] и $\mathrm{Ge} / \mathrm{Si}$ [28]. Как и ограниченные моды, эти моды характеризуются практическим отсутствием дисперсии вдоль оси, параллельной направлению роста, но в отличие от ограниченных мод, частоты этих мод остаются неизменными при изменении толщины слоев. Заметим, что возникновение интерфейсных мод зависит от поляризации колебаний: это определяется тем, как их частоты соотносятся с полосами частот, занимаемых продольными и поперечными колебаниями в объемных материалах.

Как следует из эксперимента [9,11-14], в сверхрешетках может возникать еще один тип мод, которые также относят к интерфейсным, хотя они и не являются сильно локализованными. Эти моды колебаний описывают совместные макроскопические колебания полярных оптических фононов в обоих материалах СР, при которых создаваемые ими электрические поля связаны между собой электростатическими граничными условиями. В пределе длинных волн частоты этих мод удовлетворяют условиям $d_{1} \varepsilon_{1}(\omega)+d_{2} \varepsilon_{2}(\omega)=0$ или $d_{1} \varepsilon_{2}(\omega)+d_{2} \varepsilon_{1}(\omega)=0$, где $d_{i}$ - толщина $i$-го слоя в СР, а $\varepsilon_{i}$ - его комплексная диэлектрическая проницаемость $[9,29]$. Поскольку для выполнения этих условий одна из диэлектрических проницаемостей должна быть отрицательна, эти частоты попадают в полосы остаточных лучей одного из материалов, то есть лежат между частотами TO- и LO-фононов в этом объемном материале. Эти моды легко отличить, поскольку их частоты зависят от отношения толщин слоев. Для наблюдения этих мод исследования рамановских спектров обычно проводятся в резонансных условиях [9]. В отличие от микроскопических интерфейсных мод, в которых колебания атомов локализованы на границах раздела, в колебаниях макроскопических интерфейсных мод обычно участвует существенно бо́льшее число атомов (в пределе длинных волн - все атомы в обоих слоях $[10,30])$.

Описанные результаты, полученные для сверхрешеток, оказываются полезными и для понимания колебательных спектров других квазидвумерных структур нанопластинок [31-33].

\section{2. Техника вычислений}

Расчеты проводились из первых принципов методом функционала плотности в базисе плоских волн с ис- пользованием программы ABINIT [34] в приближении локальной плотности (LDA). Оптимизированные псевдопотенциалы для атомов $\mathrm{Cd}, \mathrm{S}$ и $\mathrm{Se}$ были построены по схеме RRKJ [35] с помощью программы Opium. Максимальная энергия плоских волн в расчетах составляла $30 \mathrm{Ha}(816 \mathrm{eV})$. Интегрирование по зоне Бриллюэна проводилось на сетках Монхорста-Пака размером $8 \times 8 \times 4$ или $8 \times 8 \times 2$. Параметры решетки и равновесные положения атомов в сверхрешетках, ориентированных в направлении [001] и содержащих до 12 монослоев полупроводника, находились из условия уменьшения сил Гельмана-Фейнмана ниже $5 \cdot 10^{-6} \mathrm{Ha} / \mathrm{Bohr}(0.25 \mathrm{meV} / \AA)$ при точности вычисления полной энергии лучше $10^{-10} \mathrm{Ha}$. Фононные спектры найденных равновесных структур рассчитывались по формулам, полученным в теории возмущения DFPT аналогично [36].

$\mathrm{B}$ настоящей работе при определении характера колебательных мод мы будем анализировать дисперсию этих мод вдоль оси $\Lambda$ зоны Бриллюэна, поскольку именно вдоль оси $z$ в реальном пространстве, соответствующей этому направлению, в структуре сверхрешетки создаются сильные возмущения. При этом высокая симметрия малой группы волнового вектора на оси $\Lambda$ сохраняет подразделение колебаний на продольные и поперечные. Мы не будем пытаться установить принадлежность мод к макроскопическим интерфейсным модам поскольку для этого необходимо анализировать их свойства при ненулевых значениях поперечной компоненты волнового вектора фонона.

\section{3. Результаты расчетов и их обсуждение}

\section{1. Фононные спектры сверхрешеток}

Симметрия всех исследованных в настоящей работе сверхрешеток описывается пространственной группой $P \overline{4} m 2$, фононные моды в точке Г могут иметь симметрию $A_{1}, B_{2}$ и $E$. На рис. 1 и 2 показаны собственные векторы всех оптических мод в СР $(\mathrm{CdSe})_{6}(\mathrm{CdS})_{6}$, а также их частоты и симметрия. Как следует из рисунка, все представленные моды являются ограниченными: оптические колебания локализованы в одним из материалов СР и быстро затухают во втором материале.

Анализ дисперсионных кривых для оптических мод (рис. 3) показывает, что в направлении $\Gamma-Z$ частоты этих мод практически не зависят от волнового вектора (изменения частот мод не превышают $0.01 \mathrm{~cm}^{-1}$ ). Такое поведение соответствует существующим представлениям об ограниченных модах: они не обладают дисперсией, но их частоты зависят от толщины слоев CP. Последнее было продемонстрировано на $\mathrm{CP}(\mathrm{CdSe})_{n}(\mathrm{CdS})_{n}$ с толщиной индивидуальных слоев $n=1-4$.

Обсуждая поведение дисперсионных кривых в зоне Бриллюэна тетрагональной структуры, следует иметь в виду следующее. При переходе от точки $Г$ к оси $\Lambda$ 
соотношения совместности неприводимых представлений, описывающих симметрию нормальных колебаний, выглядят следующим образом: $A_{1} \rightarrow \Lambda_{1}, B_{2} \rightarrow \Lambda_{1}$, $E \rightarrow \Lambda_{3}+\Lambda_{4}\left(\Lambda_{3}\right.$ и $\Lambda_{4}-$ сопряженные представления,

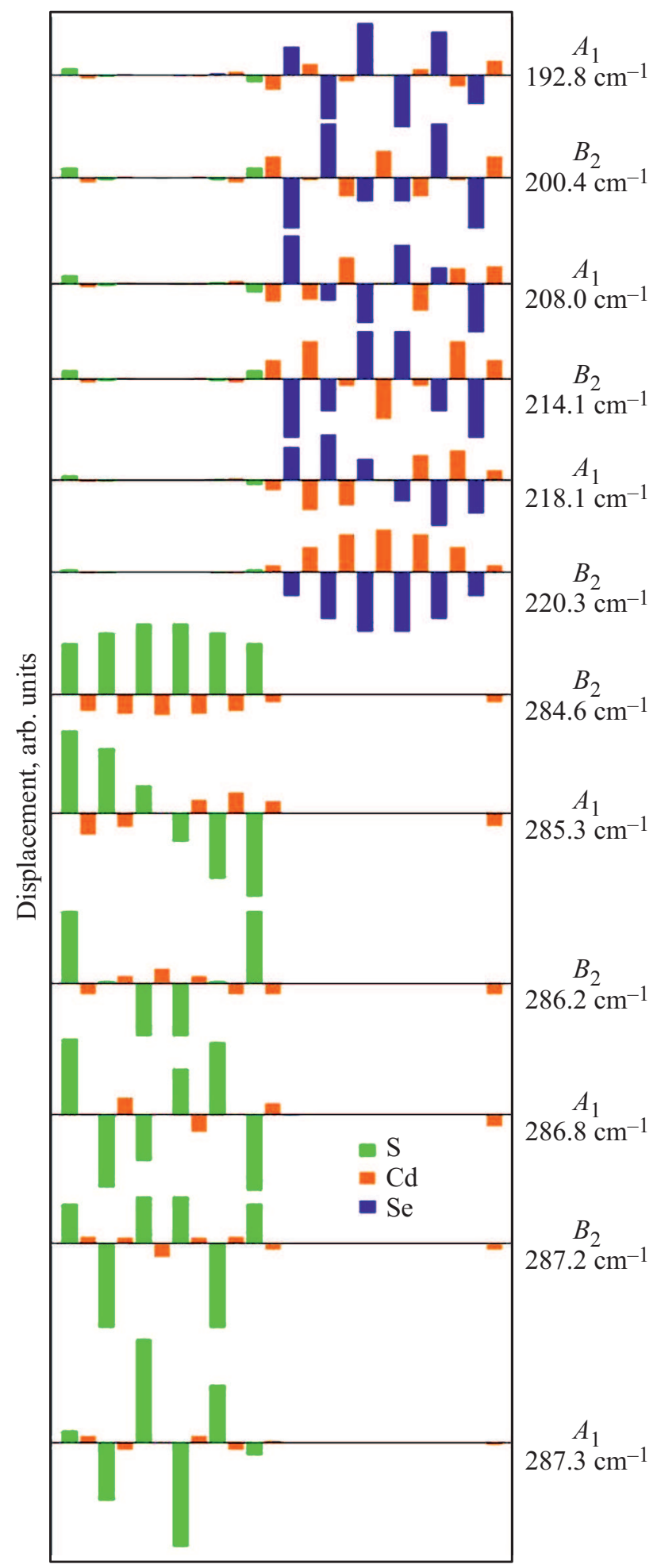

Рис. 1. Собственные векторы и частоты продольных оптических мод симметрии $A_{1}$ и $B_{2}$ при $\mathbf{q} \rightarrow 0$ в сверхрешетке $(\mathrm{CdSe})_{6}(\mathrm{CdS})_{6}$.

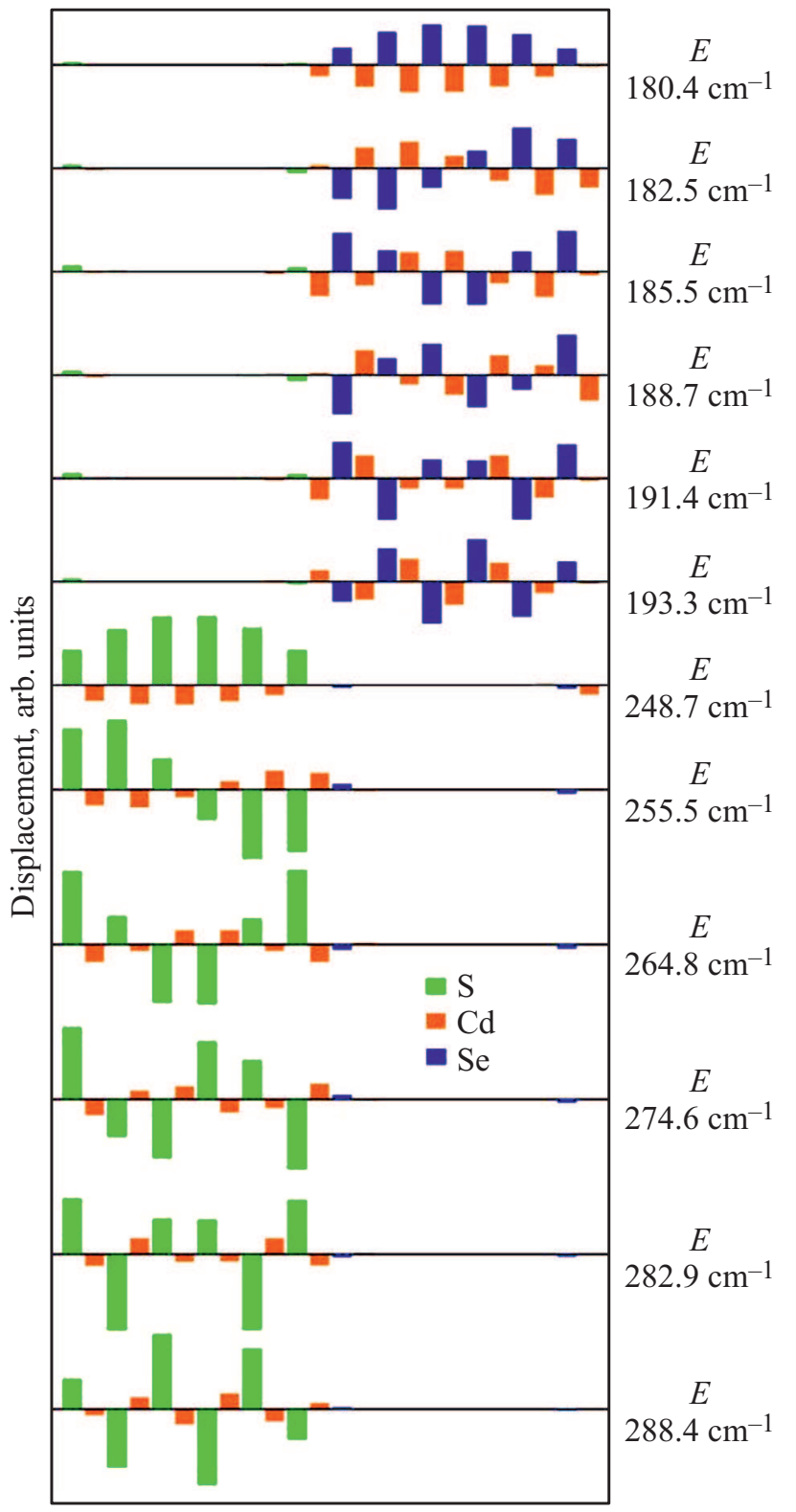

Рис. 2. Собственные векторы и частоты поперечных оптических мод симметрии $E$ при $\mathbf{q}=0$ в сверхрешетке $(\mathrm{CdSe})_{6}(\mathrm{CdS})_{6}$.

вырожденные по частоте) [37]. Таким образом, на оси $\Lambda$ перемешиваться между собой могут только продольные моды $A_{1}$ и $B_{2}$, однако это допускает перемешивание акустических и оптических мод. При переходе от точки $\Gamma$ к точкам на осях $\Delta$ и $\Sigma$ зоны Бриллюэна ситуация становится иной: соотношения совместности $A_{1} \rightarrow \Delta_{1}, B_{2} \rightarrow \Delta_{1}, E \rightarrow \Delta_{1}+\Delta_{2}$ и $A_{1} \rightarrow \Sigma_{1}, B_{2} \rightarrow \Sigma_{2}$, $E \rightarrow \Sigma_{1}+\Sigma_{2}$ допускают перемешивание продольных и поперечных колебаний. При этом на дисперсионных кривых возникает существенная дисперсия, а моды $E$ сильно расщепляются. Компонента $E$-моды, преобразующаяся по представлению $\Delta_{2}$, не перемешивается с поляризованными в направлении $z$ модами $A_{1}$ и $B_{2}$ 

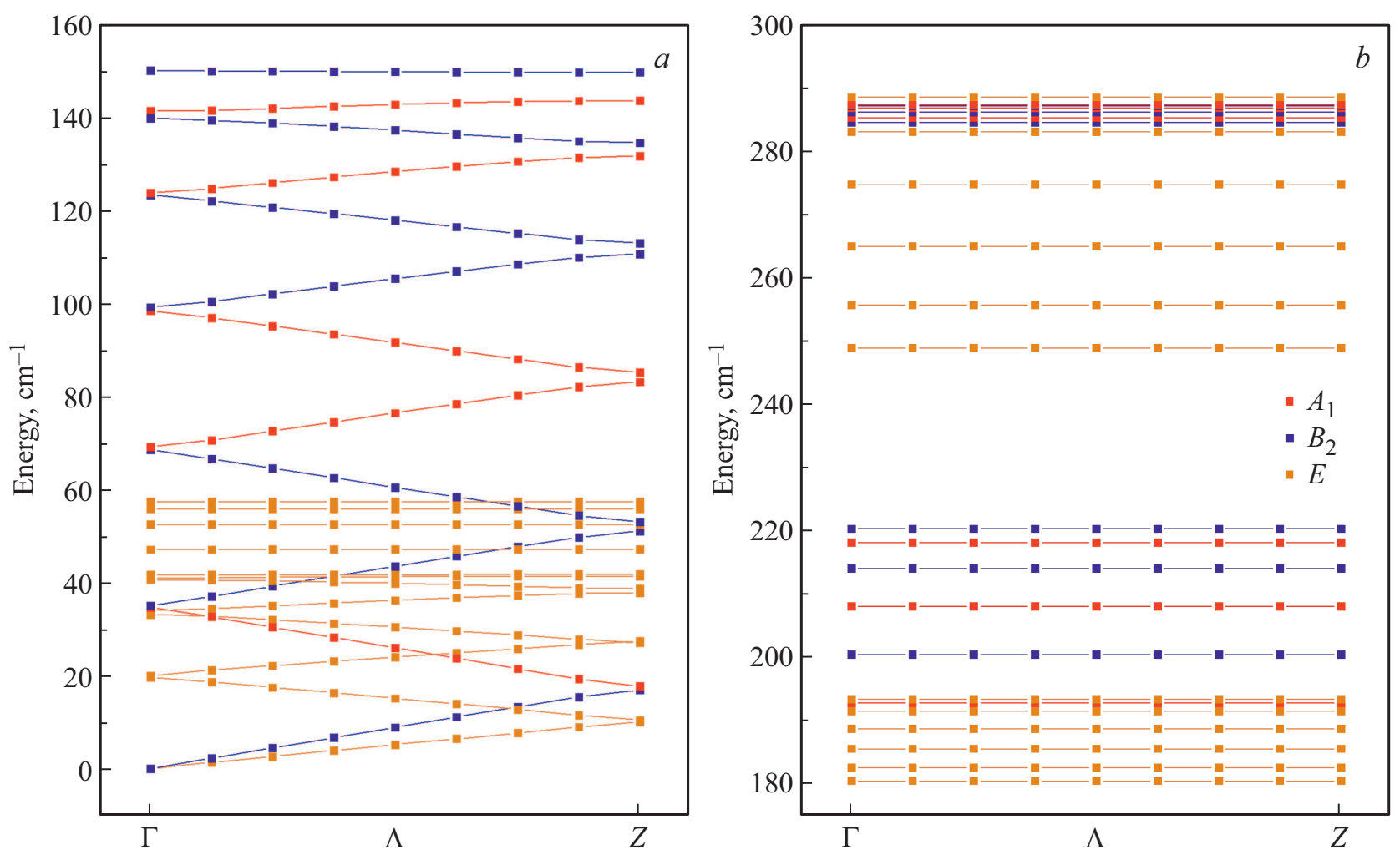

Рис. 3. Дисперсионные кривые акустических $(a)$ и оптических $(b)$ фононов в сверхрешетке $(\mathrm{CdSe})_{6}(\mathrm{CdS})_{6}$.

и поэтому ее собственный вектор описывает только смещения $\mathbf{u} \perp \mathbf{q}$ в плоскости $x y$. Мода $\Delta_{1}$, равно как и обе моды $\Sigma_{1}$ и $\Sigma_{2}$, испытывают перемешивание и демонстрируют сложную картину смещений во всех трех направлениях.

Анализ дисперсионных кривых вдоль оси $\Lambda$ в области акустических колебаний находит в этой области появление сложенных продольных и поперечных акустических мод (рис. 3) и несколько бездисперсионных ТА-мод в области $45-60 \mathrm{~cm}^{-1}$, которые мы обсудим в разд. 3.4 .

Микроскопических интерфейсных мод, собственные векторы которых были бы локализованы на границе раздела и которые бы затухали при удалении в глубь обоих материалов, в CP $\mathrm{CdSe} / \mathrm{CdS}$ не наблюдается. По-видимому, в сверхрешетках, материалы которых имеют один общий атом, этого нельзя получить в принципе, поскольку частоты потенциальных интерфейсных мод в таких СР попадают в полосы оптических колебаний по крайней мере одного из материалов СР. Однако микроскопическую интерфейсную моду можно создать искусственно, если рассмотреть свойства СР с предельно тонкими слоями одного из материалов.

\section{2. Локальная и щелевая интерфейсные моды}

Микроскопическую интерфейсную моду можно получить из ограниченной моды, если использовать в сверх- решетке слой одного из материалов минимальной толщины. Если частота этой моды окажется вне полосы оптических колебаний матрицы, то такие колебания не смогут распространяться в матрице и окажутся локализованными.

Нами был проведен поиск таких мод в СР $(\mathrm{CdSe})_{11}(\mathrm{CdS})_{1}$ и $(\mathrm{CdSe})_{1}(\mathrm{CdS})_{11}$, содержащих соответственно один монослой $\mathrm{CdS}$ в матрице $\mathrm{CdSe}$ и один монослой CdSe в матрице CdS. Как и предполагалось, в них возникают локализованные оптические моды колебаний $\mathrm{S}$ и Se. В обоих случаях введение монослоя в матрицу порождает появление трех отщепленных мод, симметрия которых $\left(B_{2}+E\right)$ совпадает с симметрией оптических фононов в объемных материалах $\left(\Gamma_{15} \rightarrow B_{2}+E\right)$. $\mathrm{B}$ матрице $\mathrm{CdSe}$ частоты колебаний слоя $\mathrm{S}$ составляют соответственно 265.2 и $274.6 \mathrm{~cm}^{-1}$, они лежат выше верхней границы полосы оптических колебаний матрицы и представляют собой локальные моды (здесь мы используем терминологию, аналогичную принятой для классификации локализованных колебаний примесей в кристаллах). В матрице $\mathrm{CdS}$ частоты колебаний слоя $\mathrm{Se}$ составляют соответственно 187.3 и $217.0 \mathrm{~cm}^{-1}$, они лежат в зазоре между полосами оптических и акустических колебаний матрицы и представляют собой щелевые моды. Собственные векторы локальной и щелевой мод показаны на рис. 4. Примесь акустических колебаний в поперечной локальной оптической моде достигает $20 \%$ в точке $Z$, а в поперечной щелевой моде $-45 \%$ 

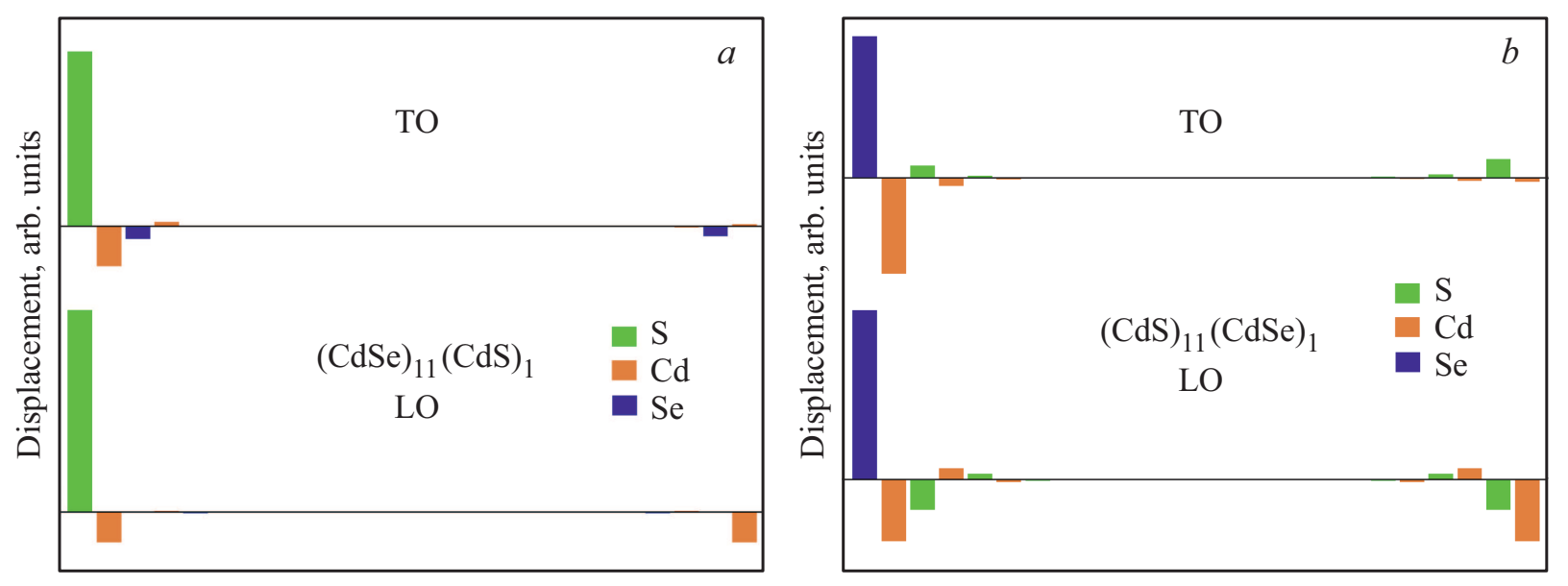

Рис. 4. Собственные векторы локальной $(a)$ и щелевой $(b)$ LO- и TO-мод при $\mathbf{q} \rightarrow 0$ в сверхрешетках $(\mathrm{CdSe})_{11}(\mathrm{CdS})_{1}$ и $(\mathrm{CdSe})_{1}(\mathrm{CdS})_{11}$.

в точке Z. В продольных локальной и щелевой модах примесь акустических колебаний не превышает $1 \%$.

Как и следовало ожидать для локальных мод, дисперсия вдоль оси $\Lambda$ как для моды $E$, так и для моды $B_{2}$ в СР $(\mathrm{CdSe})_{11}(\mathrm{CdS})_{1}$ отсутствует, а при уменьшении толщины слоя $\mathrm{CdSe}$ от 11 до 7 монослоев частоты мод слабо изменяются (соответственно на 0.16 и $0.41 \mathrm{~cm}^{-1}$ ), что целиком обусловлено небольшим (на $0.2 \%$ ) изменением параметра решетки СР в плоскости слоя. Щелевые моды также оказываются бездисперсионными, а при уменьшении числа монослоев CdS от 11 до 7 при фиксированном параметре решетки частоты мод изменяются не более, чем на $0.002 \mathrm{~cm}^{-1}$.

В то время как локальные моды известны достаточно хорошо, щелевые моды изучены менее подробно. Возможность их появления оказывается более проблематичной, поскольку для этого частота этих мод должна попадать в зазор между полосами акустических и оптических колебаний в обоих материалах CP. Для поперечных колебаний в CP CdSe/CdS верхняя граница полосы акустических колебаний лежит в районе $\sim 60 \mathrm{~cm}^{-1}$ и обсуждаемый зазор достаточно велик. Для продольных колебаний возможность появления щелевой моды определяется максимальной частотой LA-моды в точке $X$ в $\mathrm{CdS}$, которая равна $152.2 \mathrm{~cm}^{-1}$. Заметим однако, что из-за ортогональности продольных и поперечных мод всюду на оси $\Lambda$ возможность наблюдения поперечных щелевых мод оказывается шире. Так, поперечная щелевая мода $\mathrm{In}-\mathrm{Sb}$, наблюдавшаяся в $\mathrm{CP} \mathrm{GaSb} / \mathrm{InAs}$ в работе [27], оставалась бездисперсионной (то есть локализованной) несмотря на то, что она накладывалась на полосу продольных оптических фононов в одном из материалов CP (InAs).

Расчеты показывают, что вклады локализованных мод в рамановские и ИК-спектры достаточно велики, чтобы их можно было наблюдать в эксперименте.

\section{3. Зависимость частот ТО-мод от периода сверхрешетки}

В работе [33] при исследовании колебательных спектров нанопластинок $\mathrm{CdSe} / \mathrm{CdS}$ нами была обнаружена мода, связанная с ТO-колебаниями в слое $\mathrm{CdS}$, частота которой быстро уменьшалась с ростом толщины этого слоя. Эта мода давала наибольший вклад в ИК-спектры со стороны слоев $\mathrm{CdS}$. Наблюдаемый эффект был объяснен поверхностной релаксацией структуры, которая приводила к заметному укорочению межатомных расстояний $\mathrm{Cd}-\mathrm{S}$ вблизи поверхности и соответствующему возрастанию частоты колебаний. Появление $E$-моды с похожими свойствами в сверхрешетках $\mathrm{CdSe} / \mathrm{CdS}$ (нижние точки в верхнем блоке частот на рис. 5) и анализ изменения расстояний $\mathrm{Cd}-\mathrm{S}$ по сравнению с объемным $\mathrm{CdS}$ при изменении периода $\mathrm{CP}(\mathrm{CdSe})_{n}(\mathrm{CdS})_{n}$ показали, что максимальное изменение этих расстояний в CP на порядок меньше, чем в нанопластинках, а сами изменения длин связей в СР еще имеют и противоположный знак (таблица). Это может означать, что предложенный в работе [33] механизм не является единственным.

Чтобы понять причины сильного влияния толщины слоя $\mathrm{CdS}$ на частоту ТО-моды в $\mathrm{CP} \mathrm{CdSe} / \mathrm{CdS}$, мы провели анализ проекций собственных векторов колебательных мод в СР, разлагая их по ортонормированному базису нормальных колебаний LA-, LO-, TA- и TO-фононов объемного $\mathrm{CdS}$ со структурой сфалерита

$$
\mathbf{Q}_{\lambda}^{\mathrm{SL}}=\sum_{n=1}^{4} \sum_{q} C_{n q}^{\lambda} \mathbf{Q}_{n q}^{\text {bulk }},
$$

где $\lambda-$ номер колебательной моды, а $q-$ волновой вектор нормальной моды. Чтобы воспользоваться свойством ортогональности нормальных колебаний, нам следует работать с собственными векторами динамической матрицы $\mathbf{Q}_{n q}$, которые получаются путем покомпонентного умножения векторов смещения нормальных 
Частоты колебаний ТО-мод, дающих наиболее сильный вклад в ИК-спектры со стороны слоев CdS, и релаксации средней длины связи $\mathrm{Cd}-\mathrm{S}$ для нанопластинок $(\mathrm{CdSe})_{4} /(\mathrm{CdS})_{n}$ и сверхрешеток $(\mathrm{CdSe})_{n}(\mathrm{CdS})_{n}$ в зависимости от числа монослоев $n$ в слое $\mathrm{CdS}$. (Все расчеты проведены с использованием одних и тех же псевдопотенциалов)

\begin{tabular}{c|c|c|c|c}
\hline \multirow{2}{*}{$n$} & \multicolumn{2}{|c|}{ Частоты мод $\left(\mathrm{cm}^{-1}\right)$} & \multicolumn{2}{|c}{ Релаксация $R_{\mathrm{Cd}-\mathrm{S}}$} \\
\cline { 2 - 5 } & Нанопластинка & Сверхрешетка & Нанопластинка & Сверхрешетка \\
\hline \multirow{2}{*}{1} & 270.5 & 266.5 & $-0.758 \%$ & $+0.522 \%$ \\
2 & 259.8 & 257.2 & $-0.248 \%$ & $+0.485 \%$ \\
3 & 255.0 & 253.1 & $-0.114 \%$ & $+0.478 \%$ \\
4 & - & 250.9 & - & $+0.473 \%$ \\
6 & - & 248.8 & - & $+0.470 \%$
\end{tabular}

мод $\mathbf{u}_{n q}$ на квадратные корни из масс соответствующих атомов.

Базис нормальных колебаний в объемном $\mathrm{CdS}$ строился по схеме, аналогичной использованной нами в [31]. Сначала из первых принципов были рассчитаны собственные векторы LA-, LO-, TA- и TO-фононов в 13 точках зоны Бриллюэна с безразмерными волновыми векторами $0 \leq q_{z} \leq 1$, расположенными между точками $\Gamma$ $\left(q_{z}=0\right)$ и $X \quad\left(q_{z}=1\right)$ зоны Бриллюэна. Отношение амплитуд смещений атомов $\mathrm{Cd}$ и $\mathrm{S}$ для этих фононов аппроксимировалось полиномом четвертой степени от функции $\sqrt{\cos \left(\pi q_{z} / 2\right)}$ для продольных мод и функции $\cos ^{2}\left(\pi q_{z} / 2\right)$ для поперечных мод. Эти полиномы были затем использованы для построения нормированных базисных функций для произвольных значений $q z$. Для разных $q_{z}$ базисные функции ортогональны по построению. Проверка базисных функций, относящихся к одному и тому же $q z$, показала, что отклонение от их ортогональности не превышает 0.003 для всех значений $q_{z}$.

Зависимость квадратов модулей коэффициентов $C_{n q}^{\lambda}$ от $q_{z}$ для интересующих нас ТО-мод в СР показаны

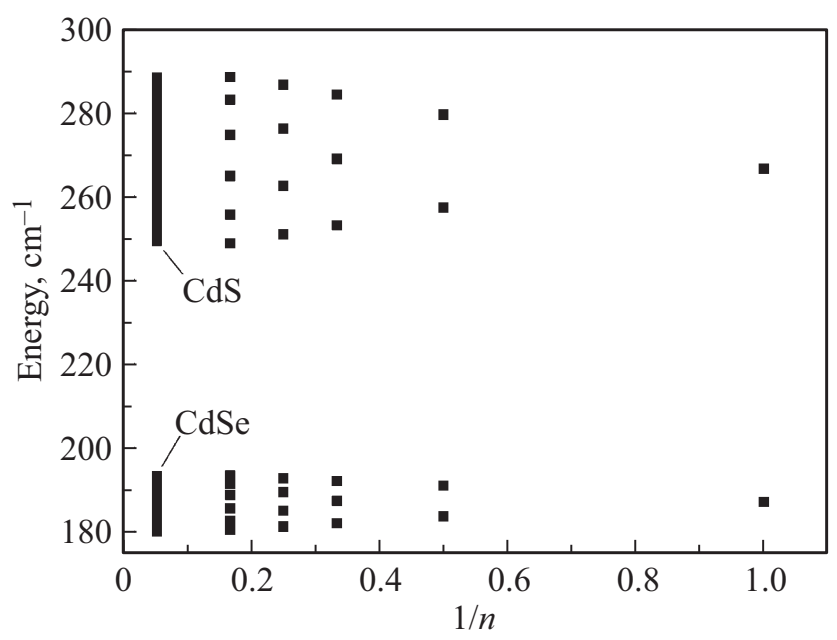

Pис. 5. Частоты ТО-мод при $\mathbf{q}=0$ в сверхрешетках $(\mathrm{CdSe})_{n}(\mathrm{CdS})_{n}$. Широкими вертикальными линиями показаны диапазоны изменения частот ТО-колебаний в бинарных соединениях CdSe и $\mathrm{CdS}$. на рис. 6,a. Наибольший вклад в картины смещений этих мод дают ТО-фононы $\mathrm{CdS}$; вклад ТА-фононов примерно в 100 раз меньше. Для ТО-моды в СР с наименьшей толщиной слоя $\mathrm{CdS}$ вклад в эту моду дают ТО-фононы из всей зоны Бриллюэна объемного $\mathrm{CdS}$, поэтому не удивительно, что частота этой моды в $\mathrm{CP}(\mathrm{CdSe})_{1}(\mathrm{CdS})_{1}$ оказывается близкой к средней частоте в полосе ТО-колебаний в объемном $\mathrm{CdS}$. C увеличением толщины слоя $\mathrm{CdS}$ во вкладах начинают преобладать фононы из все более узкого диапазона волновых векторов в окрестности точки Г, что, в соответствии с дисперсией ТО-фонона в объемном CdS, приводит к уменьшению частоты оптических колебаний.

Проекционный анализ картин смещений для обсуждаемых ТО-мод в нанопластинках $(\mathrm{CdSe})_{4} /(\mathrm{CdS})_{n}$ показывает, что диапазон волновых векторов, дающих вклад в эти моды, заметно у́же, чем в сверхрешетках $(\mathrm{CdSe})_{n}(\mathrm{CdS})_{n}$ с такой же толщиной слоя $\mathrm{CdS}$ (рис. 6). Если бы эффект размерного квантования был единственным, то частота моды в нанопластинках должна была бы быть ниже, чем в сверхрешетках. То, что в действительности наблюдается противоположный эффект (таблица), означает, что в нанопластинках имеется еще один вклад - от поверхностной релаксации, которую мы обсуждали в [33].

Для рассмотренной в п. 3.2 локальной ТО-моды в $\mathrm{CP}(\mathrm{CdSe})_{11}(\mathrm{CdS})_{1}$ проекции на TO-фононы в $\mathrm{CdS}$ (рис. $6, b$ ) оказываются очень похожими на проекции для $\mathrm{CP}(\mathrm{CdSe})_{1}(\mathrm{CdS})_{1}$, однако в этом случае вклад ТА-мод оказывается уже заметным $(21 \%$ в окрестности точки $X)$. Для локальной LO-моды в CP $(\mathrm{CdSe})_{11}(\mathrm{CdS})_{1}$ преобладающий вклад в наблюдаемые колебания дают LO-фононы CdS из окрестности точки $X$, однако то, что частота этой моды в СР $\left(274.6 \mathrm{~cm}^{-1}\right)$ оказывается намного ниже частоты LO-фонона в точке $X$ в CdS $\left(300.6 \mathrm{~cm}^{-1}\right)$, может указывать на заметный вклад $\mathrm{CdSe}$ в колебания этой моды. Наблюдаемый частотный сдвиг аналогичен сдвигу частот локальных колебаний изолированных примесей (для продольных локальных колебаний примеси $\mathrm{S}$ в матрице $\mathrm{CdSe}$ расчетная частота равна $\left.280.3 \mathrm{~cm}^{-1}\right)$. 

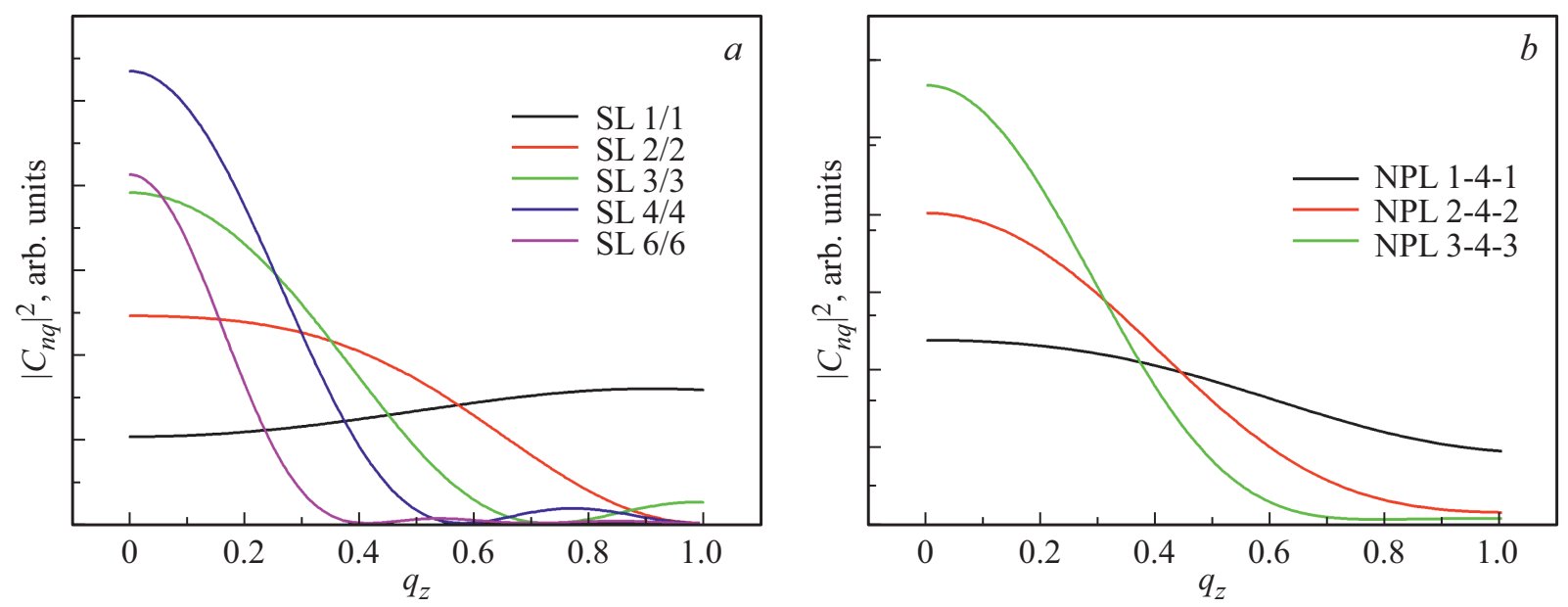

Рис. 6. Проекции собственных векторов ТО-мод, дающих наибольший вклад в ИК-спектры со стороны слоев CdS, на собственные векторы ТО-фононов в объемном $\mathrm{CdS}$ в области волновых векторов между точками $\Gamma$ и $X$ для сверхрешеток $(\mathrm{CdSe})_{n}(\mathrm{CdS})_{n}(a)$ и нанопластинок $(\mathrm{CdSe})_{4} /(\mathrm{CdS})_{n}(b)$.

\section{4. Ограниченные ТА-моды}

В принципе, возможность появления ограниченных ТА-мод возникает из-за различия спектров акустических колебаний в двух материалах сверхрешетки. Такие моды, в частности, наблюдались в CP GaSb/InAs [27], где они были связаны с локальными колебаниями пар атомов $\mathrm{Ga}-\mathrm{As}$, находящихся на границе раздела. В пользу интерфейсного характера этой моды свидетельствовала независимость ее частоты от толщины слоев СР. Аналогичных мод на границе $\mathrm{In}-\mathrm{Sb}$ не возникало, поскольку их частоты попадали в область полос акустических колебаний объемных материалов.

Анализ акустических мод в фононном спектре СР $(\mathrm{CdSe})_{6}(\mathrm{CdS})_{6}$ находит в нем, судя по собственным векторам колебаний, четыре ограниченные ТА-моды колебаний с частотами 47.9, 53.4, 56.8 и $58.3 \mathrm{~cm}^{-1}$ (рис. 7). Аналогичные моды были обнаружены в сверхрешетках $(\mathrm{CdSe})_{11}(\mathrm{CdS})_{1}$ (частота $\left.52.0 \mathrm{~cm}^{-1}\right),(\mathrm{CdSe})_{10}(\mathrm{CdS})_{2}$ (частоты 46.8 и $\left.56.6 \mathrm{~cm}^{-1}\right)$ и $(\mathrm{CdSe})_{9}(\mathrm{CdS})_{3}$ (частоты 51.7 и $\left.58.2 \mathrm{~cm}^{-1}\right)$. По оценкам, число таких мод в СР с большим периодом составляет примерно $70 \%$ от числа слоев $\mathrm{CdS}$. Расчеты дисперсии этих мод в СP $(\mathrm{CdSe})_{6}(\mathrm{CdS})_{6}$ и $(\mathrm{CdSe})_{11}(\mathrm{CdS})_{1}$ вдоль оси $\Lambda$ показывают, что моды являются бездисперсионными (изменение частоты не превышает $0.01 \mathrm{~cm}^{-1}$, рис. 3). Сравнение частот этих мод с частотами ТА-фононов в точке $X$ в объемных кристаллах $\left(45.0 \mathrm{~cm}^{-1}\right.$ в $\mathrm{CdSe}$ и $55.0 \mathrm{~cm}^{-1}$ в $\left.\mathrm{CdS}\right)$ показывает, что поперечные колебания с такими частотами действительно не могут распространяться в слоях $\mathrm{CdSe}$ и поэтому локализуются в слоях $\mathrm{CdS}$. Интересно, что частоты ряда обсуждаемых мод даже превышают частоту верхней границы акустических колебаний в объемном $\mathrm{CdS}$. Это может быть связано, во-первых, с примесью в этих акустических модах до 17-24\% оптических колебаний, а во-вторых, с увеличением частоты ТА-фононов в слое $\mathrm{CdS}$ при двуосном растяжении структуры за счет добавления слоев $\mathrm{CdSe}$.
В СР с более коротким периодом $\left((\mathrm{CdSe})_{4}(\mathrm{CdS})_{4}\right)$ сделать заключение о характере моды по собственному вектору оказывается сложнее, однако расчет дисперсии мод вдоль оси $\Lambda$ указывает на то, что мода с энергией $56.9 \mathrm{~cm}^{-1}$ является бездисперсионной, а моды с частотами 53.1 и $45.9 \mathrm{~cm}^{-1}$ имеют небольшую дисперсию

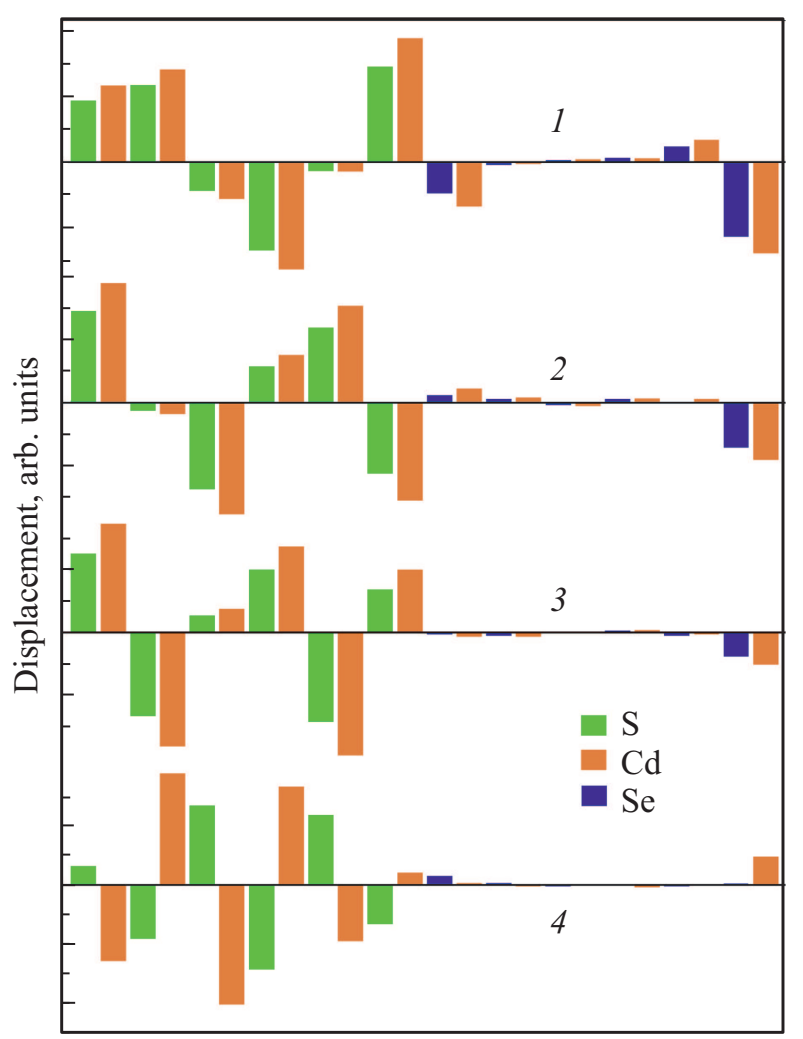

Рис. 7. Собственные векторы четырех ограниченных ТА-мод при $\mathbf{q}=0$ в сверхрешетке $(\mathrm{CdSe})_{6}(\mathrm{CdS})_{6}$ с частотами 47.9 (1), 53.4 (2) 56.8 (3) и $58.3 \mathrm{~cm}^{-1}(4)$. 

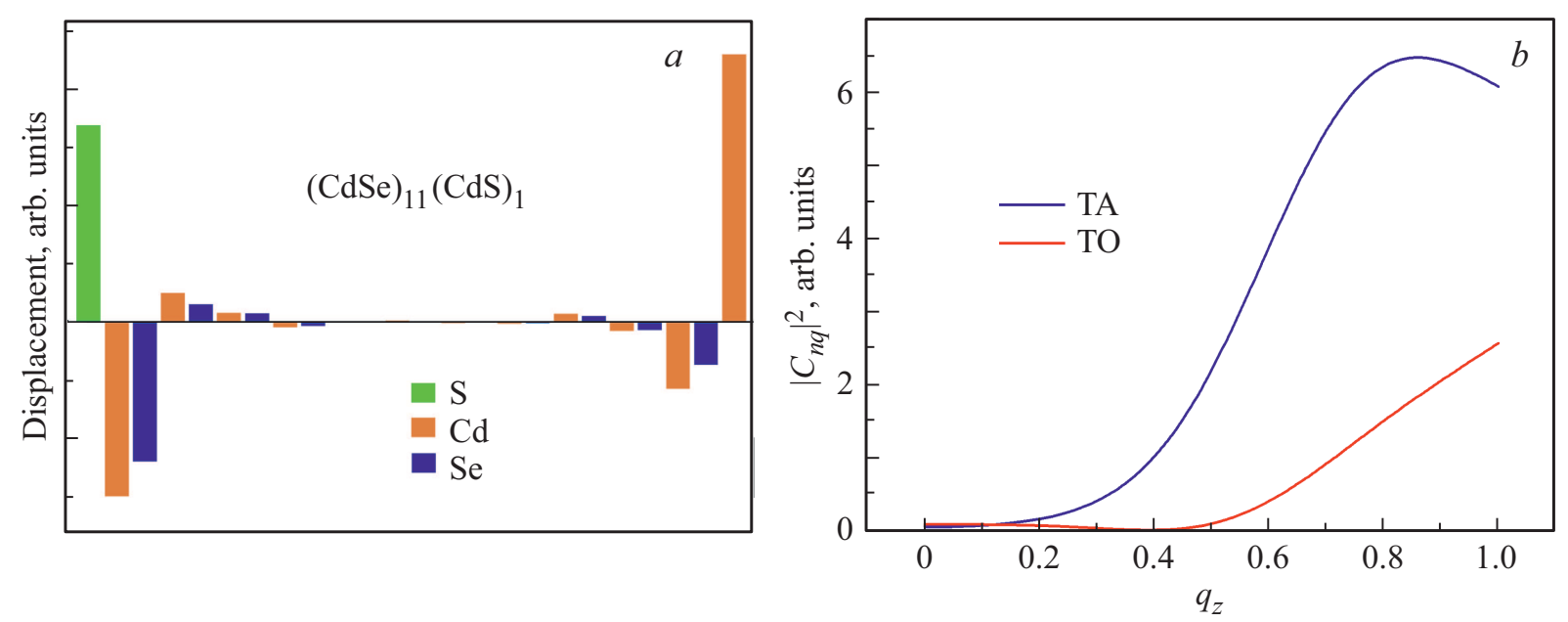

Рис. 8. $a-$ собственный вектор интерфейсной ТА-моды в сверхрешетке $(\mathrm{CdSe})_{11}(\mathrm{CdS})_{1} . b-$ проекции собственного вектора этой моды на нормальные ТА- и ТО-колебания в объемном CdS.

(0.03 и $0.13 \mathrm{~cm}^{-1}$ соответственно), что свидетельствует о слабом взаимодействии колебаний в соседних слоях $\mathrm{CdS}$ в этой CP (напомним, что скорость затухания мод возрастает по мере удалении от верхней границы акустической полосы частот в матрице CdSe, которая равна $\left.45.0 \mathrm{~cm}^{-1}\right)$. Поскольку частоты колебаний обсуждаемых ТА-мод зависят от толщины слоев, эти моды являются ограниченными, а не интерфейсными. Оценки вклада обсуждаемых ТА-мод в инфракрасные и рамановские спектры показывают, что экспериментальное наблюдение этих мод может представлять трудности, поскольку их вклад в оба типа спектров довольно невелик.

В продолжение обсуждения локализованных колебаний в СР с одним предельно тонким слоем, интересно обсудить, можно ли ограниченную ТА-моду с частотой $52.0 \mathrm{~cm}^{-1}$, возникающую в $\mathrm{CP}(\mathrm{CdSe})_{11}(\mathrm{CdS})_{1}$ (рис. 8), интерпретировать как интербейсную моду. В отличие от микроскопических интерфейсных оптических мод, особенность колебаний акустических мод состоит в том, что их собственные векторы имеют сдвоенную структуру, связанную с одновременным возникновением валентных и изгибных деформаций связей при колебаниях решетки. Авторы [27] считали похожую по свойствам ТА-моду (Ga-As в CP GaSb/InAs) интерфейсной. Как и в цитируемой работе, обсуждаемая нами мода оторвана от континуума сложенных мод, локализована и не имеет дисперсии в направлении $\Lambda$. Очень слабый $\left(\right.$ на $\left.0.17 \mathrm{~cm}^{-1}\right)$ сдвиг частоты этой моды при уменьшении толщины слоя $\mathrm{CdSe}$ в сверхрешетке от 11 до 7 монослоев целиком обусловлен изменением параметра решетки в плоскости слоев СР и подтверждает вывод об ее интерфейсном характере. По данным проекционного анализа (рис. 8, $b$ ), наибольший вклад в обсуждаемую ТА-моду дают ТА-фононы из широкой окрестности точки $X$ объемного CdS с заметной (до 30\%) примесью ТО-фононов (одинаковая симметрия этих колебаний допускает их перемешивание).

\section{4. Заключение}

В настоящей работе из первых принципов методом функционала плотности рассчитаны колебательные спектры сверхрешеток (CP) $\mathrm{CdSe} / \mathrm{CdS}$. Показано, что наряду со сложенными акустическими и ограниченными оптическими модами в СР возникает целый спектр ограниченных акустических мод, число которых составляет $\sim 70 \%$ от числа слоев материала с более высокой частотой ТА-фононов. В структурах с минимальной толщиной одного из слоев возможно формирование микроскопических интерфейсных мод типа локальной и щелевой мод. Анализ проекций собственных векторов колебательных мод в СР на ортонормированный базис нормальных колебаний в бинарных соединениях находит достаточно интенсивное перемешивание акустических и оптических колебаний даже в модах, традиционно относимых к акустическим или оптическим.

\section{Конфликт интересов}

Автор заявляет об отсутствии конфликта интересов.

\section{Список литературы}

[1] Phonons in Semiconductor Nanostructures / Eds J.-P. Leburton, J. Pascual, C.M.S. Torres. Springer, Dordrecht (1993).

[2] П. Ю, М. Кардона. Основы физики полупроводников. Физматлит, М. (2002).

[3] B. Jusserand, M. Cardona. Raman Spectroscopy of Vibrations in Superlattices. Springer, Berlin, Heidelberg (2006). Ch. 3. P. $49-152$.

[4] J.L. Merz, A.S. Barker Jr., A.C. Gossard. Appl. Phys. Lett. 31, 117 (1977).

[5] A.S. Barker, J.L. Merz, A.C. Gossard. Phys. Rev. B 17, 3181 (1978). 
[6] C. Colvard, R. Merlin, M.V. Klein, A.C. Gossard. Phys. Rev. Lett. 45, 298 (1980).

[7] F. Cerdeira, A. Pinczuk, J.C. Bean, B. Batlogg, B.A. Wilson. Appl. Phys. Lett. 45, 1138 (1984).

[8] A.K. Sood, J. Menéndez, M. Cardona, K. Ploog. Phys. Rev. Lett. 54, 2111 (1985).

[9] A.K. Sood, J. Menéndez, M. Cardona, K. Ploog. Phys. Rev. Lett. 54, 2115 (1985).

[10] C. Colvard, T.A. Gant, M.V. Klein, R. Merlin, R. Fischer, H. Morkoc, A.C. Gossard. Phys. Rev. B 31, 2080 (1985).

[11] M.V. Klein. IEEE J. Quant. Electron. QE-22, 1760 (1986).

[12] J. Menéndez. J. Lumin. 44, 285 (1989).

[13] M. Cardona. Superlat. Microstruct. 5, 27 (1989).

[14] M. Cardona. Superlat. Microstruct. 7, 183 (1990).

[15] В.А. Гайслер, А.О. Говоров, Т.В. Курочкина, Н.Т. Мошегов, С.И. Стенин, А.И. Торопов, А.П. Шебанин. ЖЭТФ 98, 1081 (1990).

[16] G. Scamarcio, L. Tapfer, W. König, A. Fischer, K. Ploog, E. Molinari, S. Baroni, P. Giannozzi, S. de Gironcoli. Phys. Rev. B 43, 14754 (1991).

[17] E. Molinari, S. Baroni, P. Giannozzi, S. de Gironcoli. Phys. Rev. B 45, 4280 (1992).

[18] B. Samson, T. Dumelow, A.A. Hamilton, T.J. Parker, S.R.P. Smith, D.R. Tilley, C.T. Foxon, D. Hilton, K.J. Moore. Phys. Rev. B 46, 2375 (1992).

[19] J. Spitzer, T. Ruf, M. Cardona, W. Dondl, R. Schorer, G. Abstreiter, E.E. Haller. Phys. Rev. Lett. 72, 1565 (1994).

[20] A. Yamamoto, T. Mishina, Y. Masumoto, M. Nakayama. Phys. Rev. Lett. 73, 740 (1994).

[21] S.P. Kozyrev, L.K. Vodopyanov. Semicond. Sci. Techniol. 14, 660 (1999).

[22] A.G. Milekhin, A.I. Nikiforov, O.P. Pchelyakov, S. Schulze, D.R.T. Zahn. Nanotechnology 13, 55 (2002).

[23] R.R. Das, Y.I. Yuzyuk, P. Bhattacharya, V. Gupta, R.S. Katiyar. Phys. Rev. B 69, 132302 (2004).

[24] А.В. Кособуцкий, Е.Н. Малышева. ФТП 42, 1227 (2008).

[25] Ю.И. Юзюк. ФТТ 54, 963 (2012).

[26] V. Davydov, E. Roginskii, Y. Kitaev, A. Smirnov, I. Eliseyev, D. Nechaev, V. Jmerik, M. Smirnov. Nanomaterials 11, 286 (2021).

[27] A. Fasolino, E. Molinari, J.C. Maan. Phys. Rev. B 33, 8889 (1986).

[28] A. Fasolino, E. Molinari. J. Phys. Colloques 48 Coll. C5, 569 (1987).

[29] R. Merlin, C. Colvard, M.V. Klein, H. Morkoç, A.Y. Cho, A.C. Gossard. Appl. Phys. Lett. 36, 43 (1980).

[30] R.E. Camley, D.L. Mills. Phys. Rev. B 29, 1695 (1984).

[31] A.I. Lebedev. Phys. Rev. B 96, 184306 (2017).

[32] S.V. Goupalov. J. Phys. Chem. C 123, 11926 (2019).

[33] A.I. Lebedev, B.M. Saidzhonov, K.A. Drozdov, A.A. Khomich, R.B. Vasiliev. J. Phys. Chem. C 125, 6758 (2021).

[34] X. Gonze, B. Amadon, P.-M. Anglade, J.-M. Beuken, F. Bottin, P. Boulanger, F. Bruneval, D. Caliste, R. Caracas, M. Côté, T. Deutsch, L. Genovese, P. Ghosez, M. Giantomassi, S. Goedecker, D.R. Hamann, P. Hermet, F. Jollet, G. Jomard, S. Leroux, M. Mancini, S. Mazevet, M.J.T. Oliveira, G. Onida, Y. Pouillon, T. Rangel, G.-M. Rignanese, D. Sangalli, R. Shaltaf, M. Torrent, M.J. Verstraete, G. Zerah, J.W. Zwanziger. Comput. Phys. Commun. 180, 2582 (2009).

[35] A.M. Rappe, K.M. Rabe, E. Kaxiras, J.D. Joannopoulos. Phys. Rev. B 41, 1227 (1990).

[36] А.И. Лебедев. ФТТ 51, 341 (2009).

[37] Bilbao Crystallographic Server http://www.cryst.ehu.es/.

Редактор Т.Н. Василевская 